\title{
Comparision Of BRAF V600E, COX-2 and p53 As Biomarkers For The Early Detection Of Colorectal Cancer
}

\author{
- - - - - - - - - - - - - Hina Wasti, Summaya Shawana ---------------
}

ABSTRACT:

Colorectal cancer (CRC) is one of the most common types of gastrointestinal cancer. Almost two million new cases of $\mathrm{CRC}$ are diagnosed every year, making $\mathrm{CRC}$ the third most common cancer and the fourth most common cancer-associated cause of mortality in the world. The onset and development of CRC is induced by a combination of genetic and environmental factors including social, cultural and lifestyle factors. Age is considered as main risk factor for the colorectal cancer, there is remarkable increase past the fifth decade of life. Because of its high incidence and mortality rate worldwide, colorectal cancer (CRC) has become a global public health problem. Patients with CRC are typically asymptomatic and therefore it is difficult to diagnose disease until advanced stages, where the disease becomes incurable. Early diagnosis and therapy is able to decrease the risk of CRC in this asymptomatic population; however, early diagnosis of CRC remains a challenge in clinical practice. This review article was a comparative study and aims to explore the ability of the selected markers for early diagnosis of colorectal cancers for long term survival. Hence, identification of novel non-invasive diagnostic methods for early tumor detection in CRC is required. Screening of average-risk individuals can reduce CRC mortality by detecting cancer at an early curable stage. There is need for the implementation of new speci?c and more sensitive biomarkers in upcoming future which will improve diagnostic strategies and allowing clinicians to detect CRC cases in the earliest stages of the disease, to improve the prognosis of thousands of patients.

Keywords: BRAF V600E, COX-2, p53, Colorectal carcinoma, Early detection.

\section{INTRODUCTION:}

Colorectal cancer is the most prevalent cancer of gastrointestinal tract globally and is the leading cause of cancer-related death. Every year around two million new cases are diagnosed all over the world. The risk of colorectal cancer increases gradually and it is related with some demographic features like age, gender, disease history and lifestyle. There is diversity in genetic predisposition for colorectal carcinoma cases. There is transition of normal mucosa into a premalignant polyp and ultimately develops in to a cancer due to certain genetic and epigenetic changes. ${ }^{1,2}$

Recent evidence suggests that one third of sporadic colorectal cancers are thought to arise from the progression of premalignant serrated lesion. ${ }^{3,4}$ There are various mutations involving the tumour suppressor genes, proto oncogenes and the genes responsible for DNA repair mechanisms. The mechanisms accountable for the pathogenesis of colorectal cancer are chromosomal instability (CIN), $\mathrm{CpG}$ islandmethylator phenotype (CIMP) and microsatellite instability (MSI). Mutations in APC, MYC, KRAS, BRAF, TP53, COX-2and TGF signaling genes are detected frequently in CRCs. ${ }^{5}$ The reported 5-year survival rate of colorectal cancer is about $90 \%$ and it can decreases up to

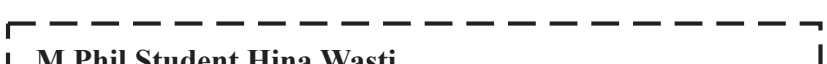

I.Phil Student Hina Wasti,

Senior Lecturer, Department of Pathology

Bahria University Medical and Dental College, Karachi

I Email: drhinafaisal@gmail.com

I Summaya Shawana,

I Associate Professor, Department of Pathology

Bahria University Medical and Dental College, Karachi

I Received: 11-02-19

Accepted: 12-03-19
$14 \%$ for the early stage of this lesion. ${ }^{6} \mathrm{CRC}$ may develops from a benign adenoma, and the estimated time for the development of adenoma into adenocarcinoma is approximated 5-10 years. ${ }^{7,8}$ At present colonoscopy is the gold- standard early diagnostic test to determine the colonic pathology. ${ }^{9,10}$ Screening colonoscopy is estimated to reduce colorectal cancer incidence by $69 \%$ and mortality by $68 \% .{ }^{11}$ As colorectal cancer is a heterogeneous disease for which chemotherapy is considered to be the backbone of treatment, specific biomarkers now have been established for detection of tumors at an early stage of disease and also to predict the treatment adequacy to improve the survival time. ${ }^{12,13}$ Some of such biomarkers include BRAF V600E, COX-2 and, P53.

BRAF (v-Raf murine sarcoma viral oncogene homolog B1) is a member of the RAS/RAF family, an which has coding an enzyme, and suggested as integral component of the cascade. BRAF oncogene has presented as direct effector of RAS and promotes the phosphorylation of MEK, which further causes tumour growth and survival. For colorectal cancer,BRAF mutations have accounts for about $8 \%$ of cases. ${ }^{14}$ At present $>95 \%$ of BRAF point mutations have been observed to at BRAF V600 in which GTG $>$ GAG substitution results in V600E amino acid change i-e the substitution of glutamate by the valine at residue $600 .{ }^{15,16}$ COX-2 has been recognized as important member of Cyclooxygenases (COXs) family which appears as an important regulator of cell proliferation. COX-2 is an inducible enzyme which accounts for the development of epithelial cell dysplasia, carcinoma, invasion and metastasis thus contributing to the development or progression of malignancy. ${ }^{17}$ 
In various studies $\mathrm{p} 53$ has been recognized as a cellular SV40 large T antigen-binding protein. ${ }^{18}$ The $\mathrm{p} 53$ signaling has been frequently impaired in CRC. p53 is a well-known tumor suppressor which promotes the transcription of various targeted genes namely $\mathrm{p} 21$ and $\mathrm{p} 27$. It also has a significant central role in cell cycle control, senescence, and apoptosis and in the prevention of cellular stress by mediating upregulation of p21 and PTEN, inhibition of AKT, and decrease of cyclin E/CDK2. ${ }^{19}$ Early detection of CRC improves the 5 years survival rate from $12-13 \%$ in stage IV metastatic disease to $90 \%$ in stage I-II early stage disease. ${ }^{20}$ This review article was a comparative study and aimed to explore the ability of the selected markers for early diagnosis of colorectal cancers for long term survival.

\section{METHODOLOGY:}

A Literature search was done by using Pubmed and Google scholar from 2009 - 2018.Key words and phrases used were ,colorectal cancer, early diagnosis, biomarkers, BRAFV600E, P53, COX-2. Multiple studies were scrutinized for the use of immunohistochemistry in early detection of colorectal cancer. 47 relevant articles are included for write up of this review article. The articles were analyzed and then composed the review article to assess the comparative analysis of these biomarkers for the early detection of colorectal cancer.

\section{LITERATURE REVIEW:}

\section{BRAFV600E:}

The BRAF oncogene is an integral component of the MAP kinase signaling pathway (RAS-RAF-MEKERK).Oncogenic activation of BRAF leads to constitutive kinase activity and phosphorylation of downstream targets of the RAS/RAF/MAPK signaling pathway.BRAF mutation constitues an alternative molecular pathway in the early carcinogenesis and accounts for $15 \%$ cases of sporadic colorectal cancer.V600E mutation is assumed an early event in serrated pathway of tumourigenesis and is greatly associated with proximal location, female gender, $\mathrm{CpG}$ island methylator phenotype and microsatellite instability (MSI). ${ }^{21,22}$

KRAS and BRAF are prime oncogenic drivers for colorectal cancer. Mutational analyses of these two important protooncogens have been a centre of research interest in recent years. ${ }^{23}$ The $B R A F \mathrm{~V} 600 \mathrm{E}$ are generally mutually exclusive with another proto-oncogene such as KRAS, both of them have been implicated in the equivalent downstream effects in tumorigenesis. Mutations of these genes might play distinct roles in tumor initiation and/or maintenance. The activating BRAFV600E mutations have been revealed to play a role in tumor invasion and evasion of apoptosis.

$B R A F$ mutations are found in $7 \%$ of cancers, with $B R A F$ V600E accounting for $>90 \%$ of mutations in $B R A F$-mutated cancers. Between $8 \%$ and $12 \%$ of metastatic CRC (mCRC) cases harbor a $B R A F$ mutation. ${ }^{24} B R A F V 600 E$ significantly increases the DNA methylation of CIMP-associated markers in primary colorectal tumors. Moreover these $B R A F$ mutations and have been observed in early precursor lesions of colorectal cancer. ${ }^{25}$ This mutation has been observed as an important predictive factor for adjuvant therapy for colorectal cancer thereby the mutation status of the tumor should be screened right before starting the treatment. The mutation status of BRAF has shown great diversity among different populations and regions. ${ }^{26}$ BRAF mutations could be considered as a stratification factor for the adjuvant therapy.For patients having MMR-deficient (dMMR) CRC, BRAFV600E mutation revealed a sporadic origin. ${ }^{27}$ Therfore both BRAF mutation and mismatch repair (MMR) statuses should be determined in all CRC to differentiate sporadic tumors from Lynch syndrome-related tumors. ${ }^{28}$

The early screening of $B R A F$ V600E might improve the evaluation of the risks for colorectal cancer and give the effective management of the patients and also important in predicting the prognosis of early CRCs. ${ }^{29} \mathrm{~A}$ study revealed BRAF mutations in $4.0 \%$ of colorectal cancers. ${ }^{30}$ Previous studies have suggested BRAF V600E mutation as an independent prognostic factor which is significantly linked with prolonged DFS (disease free survival). The association of BRAFV600E and MSI phenotype showed a better survival for earlier tumor stage. ${ }^{31}$ Various studies have proved that for (HNPCC) diagnostics, (a hereditary condition ) BRAF V600E mutation within BRAF has been proposed as a convenient, reliable, fast, and low cost strategy which simplifies genetic testing for HNPCC and therefore should be recommended for early diagnosis as it can improve the efficiency of genetictesting for HNPCC. ${ }^{32}$

At present BRAF VE1 immunohistochemistry has been identified as a useful screening tool for the detection of BRAF V600E mutation in CRCs. The BRAF VE1 IHC is more cost-effective and less time-consuming than $B R A F$ sequencing studies. ${ }^{33}$ It reveals good diagnostic performance and excellent sensitivity on IHC (sensitivity, specificity, and positive predictive values are $96.1 \%, 94 \%$, and 89.15 respectively).Moreover the BRAF V600E mutation has been appraised as an early event in colorectal cancer with multifaceted roles for progression, diagnosis and the prognosis of colorectal cancer. ${ }^{34,35}$

\section{P53:}

A well- known tumor suppressor gene p53 has been recognized as important components of our body's defense system which has been working for cancer progression control. Multiple studies have been proposed that the CRC carcinogenesis has significantly involves the mutations in various recognized proto-oncogenes namely the $K$-Ras, $A P C$ and $p 53$. Among them the p53 mutation is playing an important role in colorectal carcinogenesis. It also helps in determining the biologic basis of the disease which is implicated in the early stages of ulcerative colitis and tumorogenesis of the colorectum. ${ }^{39}$ 
This marker has been considered as a good competitor for early detection marker panel of colorectal cancer. Along with it the antibodies to p53 tumor suppressor protein have been identified as early biomarker for colorectal cancer. ${ }^{40}$

The protein expression of $\mathrm{p} 53$ in dysplastic crypts may serve as an important biomarker for colorectal cancer.0-85\% of colitis-associated cancers have defective $p 53$ gene which can be recognized via immunohistochemistry. Now a days screening method like Immunohistochemistry for the p53 biomarker in tissue samples has been considered as a useful tool for estimating the risk of morphological changes, distinction of intraepithelial neoplasms, and the progression in to malignant neoplasm involving the colonic epithelium. Also Immunohistochemistry (IHC) has been identified as a fast, most convenient and reliable method in detecting the level of p53 mutations in early precursor lesions for colorectal carcinogensis. ${ }^{41}$

The Overexpression of p53 in colonic epithelia has been identified as a most valuable tissue biomarker in surveillance of colorectal carcinogenesis. Also p 53 has been recommended for a better quantification of the risk for colon cancer. The reactivation and remodeling of p53 function has an unconventional role in colorectal carcinoma. Proper understanding of screening of this marker may allow a better stratification of early dysplastic changes and invasive carcinoma, in order to personalize treatment and surveillance. ${ }^{42}$

\section{COX -2:}

The levels of cyclo oxegenase-2 in early stages of colorectal cancer have been proposed for the early detection of CRC. ${ }^{43} \mathrm{It}$ has been recognized as a useful diagnostic marker for the CRC patients with Stage I or II disease. Cox- 2 has been found as potential blood markers and may be useful in identifying early stage CRC. ${ }^{44}$ Targeting the inhibition of COX-2 expression may help to control the progression of carcinoma, including colonic carcinoma. ${ }^{45}$

Review of numerous studies reveales that expression of COX-2 is related with some important clinicopathological parameters namely the lymphovascular invasion, serosal involvement, metastasis of multiple lymph nodes, Duke's stage, and poorly differentiated cancer. It has synergistic effects in colorectal cancer carcinogenesis. COX-2 influences different steps in cancer progression. It increases the production of prostaglandin, inhibits tumor cell apoptosis and promotes cell proliferation and tumor angiogenesis, and activates the prototype of carcinogenic substances. Significantly higher levels of COX2 in precancerous lesions and carcinoma in situ point towards an early event in tumorigenesis. ${ }^{45} \mathrm{COX}-2$ has also been recognized as a useful prognostic marker for colorectal cancer and its highest levels of expression may correspond with tumor recurrences. Routine screening of COX-2 may provide an effective index for prognosis of those at higher risk of disease metastases.
Further BRAF V600E is currently under focus as a potential prognostic and predictive biomarker which may improve assessment of colorectal cancer risk and guiding tool for patient management.

\section{CONCLUSION:}

$B R A F$ V600E, p53 and COX-2 have been recognized as markers for early detection for colorectal cancer. Amongst these markers mutated BRAFV600E is now considered as the most promising tool especially those associated with MSI.

\section{REFERENCES:}

1. Mármol I, Sánchez-de-Diego C, PradillaDieste A, Cerrada E, Rodriguez Yoldi M. Colorectal carcinoma: a general overview and future perspectives in colorectal cancer. International journal of molecular sciences. 2017;18(1):197.

2. Cancer Genome Atlas Network. Comprehensive molecular characterization of human colon and rectal cancer.Nature. 2012;487(7407):330.

3. Rex DK, Ahnen DJ, Baron JA, Batts KP, Burke CA, Burt RW, et al. Serrated Lesions of the Colorectum: Review and Recommendations From an Expert Panel. The American journal of gastroenterology. 2012. Epub 2012/06/20. https://doi.org/10.1038/ajg.2012.161 PMID: 22710576.

4. Snover DC. Update on the serrated pathway to colorectal carcinoma. Human pathology. 2011; 42(1):1- 10. Epub 2010/09/28. https://doi.org/10.1016/j.humpath.2010.06.002 PMID: 20869746.

5. Chen D et al. BRAFV600E mutation and its association with clinicopathological features of colorectal cancer: a systematic review and meta-analysis. PloS one. 2014; 9(3):e90607.

6. Nikolaou S, Qiu S, Fiorentino F, Rasheed S, Tekkis P, Kontovounisios C. Systematic review of blood diagnostic markers in colorectal cancer. Techniques in coloproctology. 2018;1:1-8.

7. Toiyama Y, Tanaka K, Inoue Y, Mohri Y, Kusunoki M. Circulating cell-free microRNAs as biomarkers for colorectal cancer. Surgery today. 2016;46(1):13-24.

8. Lin J, Chuang CC, Zuo L. Potential roles of microRNAs and ROS in colorectal cancer: diagnostic biomarkers and therapeutic targets. Oncotarget. 2017;8(10):17328.

9. Heiss JA, Brenner H. Epigenome-wide discovery and evaluation of leukocyte DNA methylation markers for the detection of colorectal cancer in a screening setting. Clinical epigenetics. 2017;9(1):24.

10. Vatandoost $\mathrm{N}$ et al. Early detection of colorectal cancer: from conventional methods to novel biomarkers. Journal of cancer research and clinical oncology. 2016;142(2):341-51.

11. Brenner H, Stock C, Hoffmeister M. Colorectal cancer screening: the time to act is now. BMC medicine. 2015;13(1):262.

12. Bupathi $\mathrm{M}, \mathrm{Wu} \mathrm{C}$. Biomarkers for immune therapy in colorectal cancer: mismatch-repair deficiency and others. Journal of gastrointestinal oncology. 2016;7(5):713.

13. Lievre A, Blons H, Laurent-Puig P. Oncogenic mutations as predictive factors in colorectal cancer. Oncogene. 2010;29(21):3033.

14. Sclafani F, Gullo G, Sheahan K, Crown J. BRAF mutations in melanoma and colorectal cancer: a single oncogenic mutation 
with different tumour phenotypes and clinical implications. Critical reviews in oncology/hematology. 2013;87(1):55-68.

15. Yuan ZX et al. The prognostic role of BRAF mutation in metastatic colorectal cancer receiving anti-EGFR monoclonal antibodies: a meta-analysis. PLoS one. 2013;8(6):e65995.

16. Thomsen CE, Appelt AL, Andersen RF, Lindebjerg J, Jensen $\mathrm{LH}$, Jakobsen A. The prognostic value of simultaneous tumor and serum RAS/RAF mutations in localized colon cancer.Cancer medicine. 2017;6(5):928-36.

17. Roelofs HM, TeMorsche RH, van Heumen BW, Nagengast FM, Peters WH. Over-expression of COX-2 mRNA in colorectal cancer. BMC gastroenterology. 2014;14(1):90

18. Farnebo M, Bykov VJ, Wiman KG. The p53 tumor suppressor: a master regulator of diverse cellular processes and therapeutic target in cancer. Biochemical and biophysical research communications. 2010;396(1):85-9.

19. Li XL, Zhou J, Chen ZR, Chng WJ. P53 mutations in colorectal cancer-molecular pathogenesis and pharmacological reactivation. World journal of gastroenterology: WJG. 2015;21(1):84.

20. Hao YX et al. KRAS and BRAF mutations in serum exosomes from patients with colorectal cancer in a Chinese population.Oncology letters. 2017;13(5):3608-16.

21. Hinoue $T$ et al. Analysis of the association between CIMP and BRAFV600E in colorectal cancer by DNA methylation profiling.PloS one. 2009;4(12):e8357.

22. Bond CE, Whitehall VL. How the BRAF V600E Mutation Defines a Distinct Subgroup of Colorectal Cancer: Molecular and Clinical Implications. Gastroenterology research and practice. 2018;2018

23. Tan $\mathrm{C}, \mathrm{Du} \mathrm{X}$. KRAS mutation testing in metastatic colorectal cancer. World journal of gastroenterology: WJG. 2012;18(37):5171

24. Sanz-Garcia E, Argiles G, Elez E, Tabernero J. BRAF mutant colorectal cancer: prognosis, treatment, and new perspectives. Annals of Oncology. 2017;28(11):2648-57.

25. Khan SA et al. V600E BRAF mutations are alternative early molecular events in a subset of KIT/PDGFRA wild-type gastrointestinal stromal tumours. Journal of clinical pathology. 2009;62(7):613-6.

26. Mostert B et al. KRAS and BRAF mutation status in circulating colorectal tumor cells and their correlation with primary and metastatic tumor tissue. International journal of cancer. 2013;133(1):130-41

27. Ye JX, Liu Y, Qin Y, Zhong HH, Yi WN, Shi XY. KRAS and BRAF gene mutations and DNA mismatch repair status in Chinese colorectal carcinoma patients. World journal of gastroenterology: WJG. 2015;21(5):1595.

28. Cohen R, Cervera P, Svrcek M, Pellat A, Dreyer C, de Gramont A, Andre T. BRAF-mutated colorectal cancer: what is the optimal strategy for treatment?. Current treatment options in oncology. 2017;18(2):9.

29. Mesteri I, Bayer G, Meyer J, Capper D, Schoppmann SF, Von Deimling A, Birner P. Improved molecular classification of serrated lesions of the colon by immunohistochemical detection of BRAF V600E. Modern Pathology. 2014;27(1):135.

30. Won DD, Im Lee J, Lee IK, Oh ST, Jung ES, Lee SH. The prognostic significance of KRAS and BRAF mutation status in Korean colorectal cancer patients.BMC cancer. 2017;17(1):403.
31. Yaeger R et al. BRAF mutation predicts for poor outcomes after metastasectomy in patients with metastatic colorectal cancer. Cancer. 2014;120(15):2316-24.

32. Domingo E, Laiho P, Ollikainen M, Pinto M, Wang L, French AJ, Westra J, Frebourg T, Espin E, Armengol M, Hamelin R. BRAF screening as a low-cost effective strategy for simplifying HNPCC genetic testing. Journal of medical genetics. 2004;41(9):664-8.

33. Kwon JH, Jeong BK, Yoon YS, Yu CS, Kim J. Utility of BRAF VE1 Immunohistochemistry as a Screening Tool for Colorectal Cancer Harboring BRAF V600E Mutation. Journal of pathology and translational medicine. 2018;52(3):157.

34. Schafroth $\mathrm{C}$ et al. VE1 immunohistochemistry predicts BRAF V600E mutation status and clinical outcome in colorectal cancer. Oncotarget. 2015;6(39):41453

35. 1. Tie J, Desai J. Targeting BRAF mutant metastatic colorectal cancer: clinical implications and emerging therapeutic strategies. Targeted oncology. 2015;10:179-188.

36. Levine AJ, Oren M. The first 30 years of $\mathrm{p} 53$ : growing ever more complex. Nat Rev Cancer. 2009;9:749-758.

37. Cottu PH et al. Inverse correlation between RER+ status and p53 mutation in colorectal cancer cell lines. Oncogene. 1996;13:2727-2730.

38. López I, P Oliveira L, Tucci P, Alvarez-Valín F, A Coudry R, Marín M. Different mutation profiles associated to P53 accumulation in colorectal cancer. Gene. 2012;499:81-87.

39. Lu HY, Lin RT, Zhou GX, Yu TM, Liu ZJ. Critical Role of p53 and K-ras in the Diagnosis of Early Colorectal Cancer: a One-year, Single-center Analysis. International journal of medical sciences. 2017;14(11):1154.

40. Teras LR, Gapstur SM, Maliniak ML, Jacobs EJ, Gansler T, Michel A, Pawlita M, Waterboer T, Campbell PT. Prediagnostic antibodies to serum p53 and subsequent colorectal cancer. Cancer Epidemiology and Prevention Biomarkers. 2018;27(2):219-23.

41. Kobayashi K, Tomita H, Shimizu M, Tanaka T, Suzui N, Miyazaki T, Hara A. p53 expression as a diagnostic biomarker in ulcerative colitis-associated cancer. International journal of molecular sciences. 2017;18(6):1284.

42. C, Nichita L, Voiosu T, Bastian A, Cioplea M, Micu G, Pop G, Sticlaru L, Bengus A, Voiosu A, Mateescu RB. Expression profile of p53 and p21 in large bowel mucosa as biomarkers of inflammatory-related carcinogenesis in ulcerative colitis.Disease markers. 2016.

43. Tatsu K, Hayashi S, Shimada I, Matsui K. Cyclooxygenase2 in sporadic colorectal polyps: immunohistochemical study and its importance in the early stages of colorectal tumorigenesis. Pathology-Research and Practice. 2005;201(6):427-33.

44. Bujanda L, Sarasqueta C, Cosme A, Hijona E, EnríquezNavascués JM, Placer C, Villarreal E, Herreros-Villanueva M, Giraldez MD, Gironella M, Balaguer F. Evaluation of alpha 1-antitrypsin and the levels of mRNA expression of matrix metalloproteinase 7, urokinase type plasminogen activator receptor and COX-2 for the diagnosis of colorectal cancer. PloS one. 2013;8(1):e51810.

45. Shi G, Li D, Fu J, Sun Y, Li Y, Qu R, Jin X, Li D. Upregulation of cyclooxygenase- 2 is associated with activation of the alternative nuclear factor kappa B signaling pathway in colonic adenocarcinoma. American journal of translational research. 2015;7(9):1612. 